\title{
Unchanged thermopower enhancement at the semiconductor-metal transition in correlated $\mathrm{FeSb}_{2-x} \mathrm{Te}_{x}$
}

\author{
P. Sun, ${ }^{1, a)}$ M. Søndergaard, ${ }^{2}$ Y. Sun, ${ }^{2}$ S. Johnsen, ${ }^{2}$ B. B. Iversen, ${ }^{2}$ and F. Steglich ${ }^{1}$ \\ ${ }^{1}$ Max Planck Institute for Chemical Physics of Solids, D-01187 Dresden, Germany \\ ${ }^{2}$ Department of Chemistry, University of Aarhus, DK-8000 Aarhus C, Denmark
}

(Received 1 December 2010; accepted 29 January 2011; published online 16 February 2011)

\begin{abstract}
Substitution of $\mathrm{Sb}$ in $\mathrm{FeSb}_{2}$ by less than $0.5 \%$ of Te induces a transition from a correlated semiconductor to an unconventional metal with large effective charge carrier mass $m^{*}$. Spanning the entire range of the semiconductor-metal crossover, we observed an almost constant enhancement of the measured thermopower compared to that estimated by the classical theory of electron diffusion. Using the latter for a quantitative description one has to employ an enhancement factor of 10-30. Our observations point to the importance of electron-electron correlations in the thermal transport of $\mathrm{FeSb}_{2}$, and suggest a route to design thermoelectric materials for cryogenic applications. (C) 2011 American Institute of Physics. [doi:10.1063/1.3556645]
\end{abstract}

Current material science has not yet found efficient thermoelectric (TE) materials for cryogenic $(T<100 \mathrm{~K})$ applications such as spot cooling microelectronic superconducting devices. ${ }^{1}$ In this context recent observations of a colossal thermopower $S$ of $-(6-45) \mathrm{mV} / \mathrm{K}$ below $30 \mathrm{~K}$ in $\mathrm{FeSb}_{2}$ have stimulated a great interest in the underlying physics and also practical applications. ${ }^{2-4}$ Original interest in this compound stems from its narrow energy gap and strong electronic correlations as well as the thus induced unusual physical properties. ${ }^{5-8}$ For a long time, systems with electron correlations have been expected to be candidates for TE applications. ${ }^{1}$ Following this line are correlated semiconductors, e.g., $\mathrm{FeSi}$, and correlated metals, such as $\mathrm{CePd}_{3}$, which do show promising TE properties at cryogenic range. ${ }^{9}$ Though the figure of merit $z T\left(=T S^{2} / \rho \kappa\right.$, with $\rho$ being the electrical resistivity and $\kappa$ the thermal conductivity) is only $\sim 0.01$ at $50 \mathrm{~K}$ for $\mathrm{FeSi}$, it can be enhanced to slightly below 0.1 at $100 \mathrm{~K}$ by $5 \% \mathrm{Ir}$ doping. ${ }^{9} \mathrm{CePd}_{3}$, on the other hand, an intermediate-valence metal with low carrier density, exhibits a $z T=0.23$ at around $200 \mathrm{~K} .{ }^{9}$ Our intensive investigations on $\mathrm{FeSb}_{2}$ have already clarified the interplay between the enhanced thermopower and electron correlations, ${ }^{2,3,10,11}$ nevertheless the microscopic mechanism of the enhancement is far from being understood. ${ }^{4}$

Here, we report TE properties of $\mathrm{FeSb}_{2-x} \mathrm{Te}_{x}$ with a narrow doping range, $0<x<0.16$, where a semiconductormetal (SM) transition occurs. Magnetism and electrical resistivity over a wide $x$ range $(0-1.2)$ have already been reported by $\mathrm{Hu}$ et al. ${ }^{12}$ Our emphasis is aimed at learning to what extent the enhanced $S$ in a correlated semiconductor can be kept when crossing a SM transition. $\mathrm{FeSb}_{2}$ and $\mathrm{FeTe}_{2}$ are isostructural (marcasite type) semiconductors having rather different energy gaps. The former shows a transport gap of $E_{g}=26-36 \mathrm{meV}$ together with an even smaller one of $\sim 6 \mathrm{meV},{ }^{11}$ contrasting to a much larger $E_{g}$ of the latter, $0.2-0.5 \mathrm{eV}{ }^{13}$ The most striking finding of our study is that the enhancement to $S(T)$ (by a factor of 10-30) relative to the classical expectation, is rather robust against doping, spanning the entire range of the SM transition. This feature provides a convincing link between the enhanced $S(T)$ and the

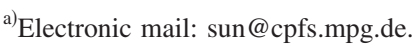

particular electronic structure of $\mathrm{FeSb}_{2}$. Upon Te doping, a large decrease in $\kappa$ is also observed which however, results in an only minor rise in $z T$, because of an overwhelming lattice contribution $\kappa_{L}$ even in the metallic regime.

Single crystals of $\mathrm{FeSb}_{2-x} \mathrm{Te}_{x}$ were grown by chemical vapor transport using $\mathrm{Br}_{2}$ as transport agent, and subsequently characterized and oriented using power x-ray and Laue diffraction methods. ${ }^{11}$ It turns out that the actual concentration $x_{a}$ of Te as estimated by Hall-effect measurements may considerably deviate from the nominal value $x_{n}$ (inset of Fig. 1); even different crystals from the same batch show slightly differing values of $x_{a}$. The reasons for this may involve the hard-to-control amount of the transport gas and different partial pressures of the formed bromides. The data presented here were obtained by employing a physical property measurement system (PPMS, Quantum Design), except for those of $x_{a}=0.01$, which has a smaller dimension $(<4 \mathrm{~mm}$ long) and was measured using a home-made cryostat equipped with a $\mathrm{Au}(\mathrm{Fe} 0.07 \%)$-chromel thermocouple. We paid particular attention to measure all the transport

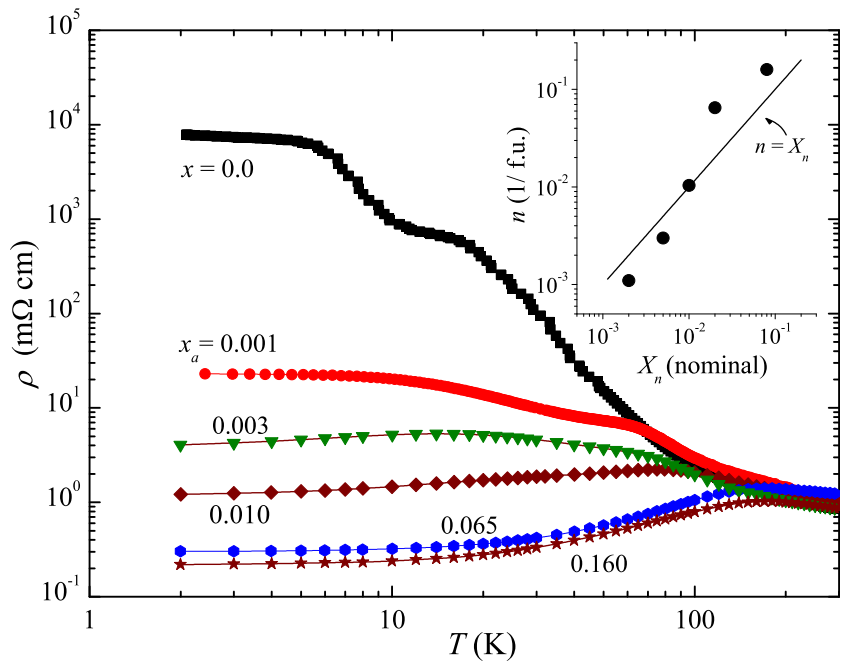

FIG. 1. (Color online) Electrical resistivity $\rho(T)$ of $\mathrm{FeSb}_{2-x} \mathrm{Te}_{x}$ with varying actual doping concentration $\left(x_{a}\right)$. Inset: correlation between carrier concentration $n$ and nominal Te content $x_{n}$, from which $x_{a}$ is determined. 


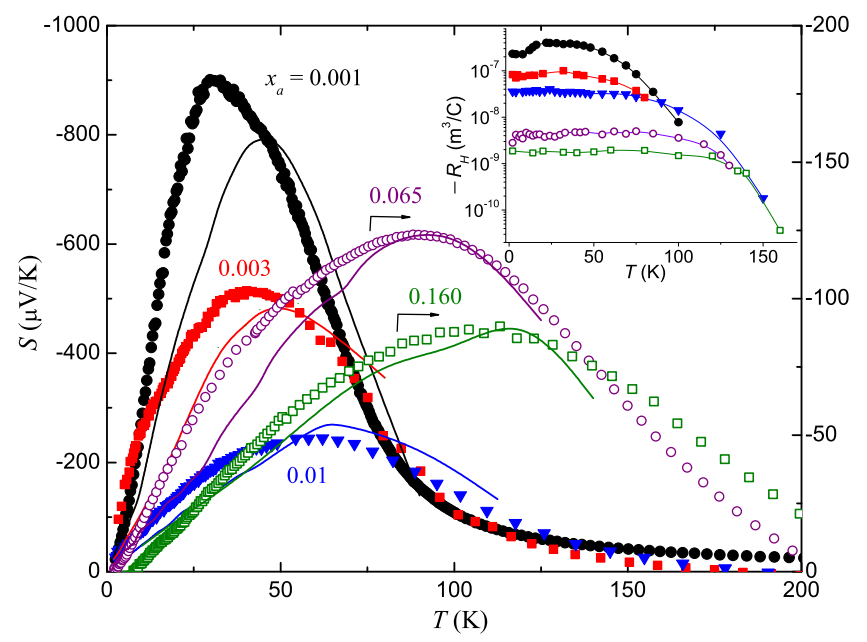

FIG. 2. (Color online) Thermopower $S(T)$ of $\mathrm{FeSb}_{2-x} \mathrm{Te}_{x}$ with varying $x_{a}$. Solid lines are theoretical calculations based on the classical formula (see text) and further enhancement factors, i.e., 18, 22, 20, 28, and 32 for $x_{a}$ $=0.001,0.003,0.01,0.065$, and 0.160, respectively. Inset: Hall coefficient $-R_{H}(T)$ for $\mathrm{FeSb}_{2-x} \mathrm{Te}_{x}$ with symbols the same as in the main panel.

properties on exactly the same crystal for each doping concentration.

The transition from a semiconducting to a metallic ground state at around $x_{a}=0.003$ is shown explicitly in Fig. 1 by the evolution of $\rho(T)$ with $x_{a}$. Upon $0.5 \%\left(x_{a}\right.$ $=0.01)$ Te doping, $\rho(T)$ at below $10 \mathrm{~K}$ is reduced by four orders of magnitude. Given the small energy gap of $\mathrm{FeSb}_{2}$ $(\sim 6 \mathrm{meV})$, the SM transition to occur at an even smaller value of $x_{a}$ is not really surprising. Above $150 \mathrm{~K}$, by contrast, $\rho(T)$ is insensitive against doping. In this temperature range, the Arrhenius function holds true up to at least $x_{a}=0.01$ with a larger transport gap of $20-40 \mathrm{meV}$, equivalent to that of pure $\mathrm{FeSb}_{2}{ }^{2,11}$ The Hall coefficient $R_{H}$ (inset of Fig. 2) is roughly constant at low temperatures for all the metallic samples from which, within a one-band model, the carrier concentration $n\left(=1 / e\left|R_{H}\right|\right)$ can be estimated (inset of Fig. 1). An anisotropic electrical transport in $\mathrm{FeSb}_{2-x} \mathrm{Te}_{x}$ was reported by Hu et al. ${ }^{12}$ This, however, is much weaker than the effect of the carrier concentration differing from sample to sample and will, therefore, be not addressed. For completeness, we list the directions of the applied heat/electrical current for all the samples employed: $\langle 001\rangle\left(\mathrm{FeSb}_{2}\right),\langle 110\rangle$ $(0.001),\langle 001\rangle(0.003),\langle 110\rangle(0.01),\langle 100\rangle(0.065)$, and $\langle 010\rangle$ $(0.160)$.

As seen in Fig. 2, the maximum absolute thermopower $\left|S_{\max }\right|$ is diminished greatly upon increasing $x_{a} \cdot\left|S_{\max }\right|$ decreases from $6-45 \mathrm{mV} / \mathrm{K}$ at around $10 \mathrm{~K}$ in nominally pure $\mathrm{FeSb}_{2}$ (Refs. 2, 3, and 11) to $0.9 \mathrm{mV} / \mathrm{K}$ at $30 \mathrm{~K}$ for $x_{a}$ $=0.001$ and eventually to $90 \mu \mathrm{V} / \mathrm{K}$ at $110 \mathrm{~K}$ for $x_{a}=0.16$, the metallic end member of this alloy series. Such a decrease in $\left|S_{\max }\right|$ is not inconsistent with expectation, because the thermopower measures the energy derivative at the Fermi level of the electrical conductivity, which shows change by several orders of magnitude with Te doping (to a first order approximation, $S \sim \ln \rho$ ). However, here we put strong emphasis on one fact: by taking into account the respective carrier concentration $n$ determined from Hall coefficient (inset of Fig. 2), the large enhancement of $S(T)$ as measured compared to classical expectations ${ }^{3}$ still holds true when crossing the SM transition. In a parabolic band approxima-

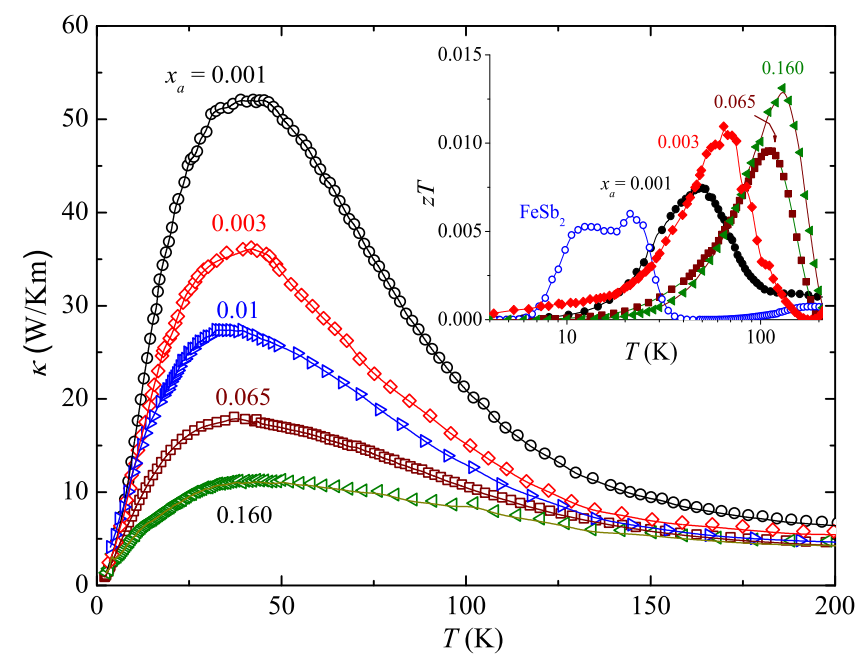

FIG. 3. (Color online) Thermal conductivity $\kappa(T)$ of $\mathrm{FeSb}_{2-x} \mathrm{Te}$ with varying $x_{a}$. Solid lines indicate lattice contribution $\kappa_{L}(T)$ to each system. Inset: $z T$ vs $T$ for the different samples.

tion, $S(T)$ of a degenerate electron system with dominant scattering by acoustic phonons is given by

$$
S(T)=\frac{\pi^{2}}{3} \frac{k_{B}}{e} \frac{k_{B} T}{\epsilon_{F}}
$$

where the Fermi energy $\epsilon_{F}$ is a function of $n$ and $m^{*}$,

$$
\epsilon_{F}=\frac{h^{2}}{2 m^{*}}\left(\frac{3 n}{8 \pi}\right)^{2 / 3} \text {. }
$$

What is remarkable is that by assuming $m^{*}=m_{0}$, the free electron mass, an almost universal factor $10-30$ has to be multiplied on Eq. (1) in order to reproduce the experimental results of all the Te doped systems, as indicated by the solidline curves (cf. Fig. 2 and its caption).

For simplicity, the above calculations were carried out up to only $100-150 \mathrm{~K}$ where $R_{H}$ is negative, whereas at higher temperatures multiband corrections have to be considered. The enhancement to $S(T)$, on the other hand, can be regarded as corresponding reduction in $\epsilon_{F}$ : for example in the case of $x_{a}=0.065, \epsilon_{F}$ estimated from Eq. (1) (when applied to describe $S(T))$ is $\sim 180 \mathrm{~K}$, a factor of 28 smaller than $\epsilon_{F}$ $=5000 \mathrm{~K}$ as estimated from the measured value of $n$ with the aid of Eq. (2). Considerable deviations between the calculated lines and the measured $S(T)$ values are observed, particularly at temperatures below that of $\left|S_{\max }\right|$. This is not too surprising, noting that the effects due to phonon drag, an additional impurity band as seen in $\mathrm{FeSb}_{2}$ (Ref. 3), as well as a possible nonparabolicity of the bands were ignored in the simple calculations. Instead of aiming for a good fitting to the experimental results, our emphasis is to understand whether and, if so, to what extent the aforedescribed thermopower is affected when crossing the SM transition.

Our observations can provide valuable insight into the unusual TE transport of $\mathrm{FeSb}_{2}$ : the enhancement to thermopower found both in the semiconducting and metallic regimes most probably has a common microscopic origin, and should be considered an intrinsic electronic property. Arguments against a dominating phonon drag effect, in addition to the reasonable fitting of $S(T)$ as shown above, are the very different evolutions of $S(T)$ and $\kappa(T)$ (Fig. 3) with $x_{a}$. While the values of both quantities are reduced, the maximum of 
the former shifts rapidly to higher temperature whereas that of the latter remains robust. The factor 10-30 of the thermopower enhancement is too large to be explained by the local-density-approximation band-structure calculations. ${ }^{8} \mathrm{~A}$ realistic scenario is to take into account a renormalization of $m^{*}$ by electron-electron correlations, similar to what occurs in heavy-fermion systems. ${ }^{14}$ This argument has found support from thermodynamic and magnetic measurements, for example, (i) the electronic specific heat (not shown here) in combination with the measured carrier concentration yields an enhanced $m^{*}$ by a similar factor $10-30$ for all the samples investigated; (ii) the magnetic susceptibility above $100 \mathrm{~K}$ persists to be thermally activated when crossing the SM transition, indicating that the narrow gap and the correlated nature do not change.

Given the importance of electron-electron correlations in the enhancement of $S(T)$, the underlying physics is yet to be clarified. Prevalent understanding is based either on local spin fluctuations as known for Kondo insulators or a nearly ferromagnetic semiconductor picture in an itinerant model. ${ }^{6}$ In particular, for pure $\mathrm{FeSb}_{2}$, where the charge carriers are assumed to be nondegenerate, our previous study ${ }^{3}$ revealed that its thermopower can be described by using not only the nondegenerate approximation, but also the degenerate approximation as given by Eq. (1), with a similar enhancement factor. Of course, it remains to be shown whether the above interpretation of the enhanced thermopower based on a strongly renormalized $m^{*}$ can be applied to pure $\mathrm{FeSb}_{2}$. Also, the observed maximum thermopower $\left|S_{\max }\right|$ for $x_{a}=0.001$ and 0.003 exceeds $\pi^{2} / 3 k_{B} / e \approx 283 \mu \mathrm{V} / \mathrm{K}$, i.e., the classical upper bound expected from Eq. (1) for degenerate electrons when $\epsilon_{F}>k_{B} T$, indicating that they might fall into the regime on the degenerate-nondegenerate borderline as well. Theoretical treatment of electron correlations in a nondegenerate approximation, however, is still an open issue. ${ }^{4}$

In view of the thermopower enhancement being almost unaffected upon crossing the SM transition, it is of great technological interest to see to which extent $\kappa$ can be reduced. As displayed in Fig. 3, there is a significant reduction of $\kappa(T)$ with increasing $x_{a}$. We attribute this reduction mainly to the introduced charge carriers rather than to chemical disorder: a small number of charge carriers in a pure semiconductor can dramatically reduce $\kappa$ as is known in, e.g., Ge. ${ }^{15}$ What is certainly unfavorable for TE application is that, in the whole doping range, the lattice contribution $\kappa_{L}$ (Ref. 16) as indicated by lines in Fig. 3, is nearly equivalent to the measured $\kappa$. The electronic portion, $\kappa_{e}$, is less than $3 \%$ of $\kappa$ at $T \leq 100 \mathrm{~K}$ for not only the semiconducting samples but also the metallic ones. As a result, $z T$ shows only moderate increase, from 0.006 in $\mathrm{FeSb}_{2}$ to 0.013 in $x_{a}=0.160$ (inset of
Fig. 3), a value similar to that of the undoped FeSi. ${ }^{9}$ Practical TE materials are those with optimized $\kappa_{L}$ that is preferably less than or at least comparable to $\kappa_{e}$. As far as $\kappa_{L}$ dominates $\kappa$, as is currently observed, it turns out to be hard to realize a sufficiently large $z T$. Further nanoprocessing to reduce $\kappa_{L}$ seems to be inevitable for possible application of this material. The preparation of thin film ${ }^{17,18}$ has already been initiated, however, well-controlled composition and purity have yet to be realized.

To summarize, we have found that the large thermopower enhancement relative to the classical expectation previously observed for correlated $\mathrm{FeSb}_{2}$ persists when crossing the SM transition induced by Te doping. In at least the metallic range, this phenomenon can be captured by the largely renormalized charge carrier mass due to electronelectron correlations. Because of a dominant lattice thermal conductivity, $z T$ remains low in the $\mathrm{FeSb}_{2-x} \mathrm{Te}_{x}$ system. However, our study emphasizes the potential in exploring unconventional TE materials through tuning a correlated system between semiconducting and metallic regimes. Along this line, searching for new correlated semiconductors with intrinsically low thermal conductivity (complex lattice structure, heavy atoms) is badly called for.

${ }^{1}$ G. D. Mahan, Solid State Phys. 51, 81 (1997).

${ }^{2}$ A. Bentien, S. Johnsen, G. K. H. Madsen, B. B. Iversen, and F. Steglich, Europhys. Lett. 80, 17008 (2007).

${ }^{3}$ P. Sun, N. Oeschler, S. Johnsen, B. B. Iversen, and F. Steglich, Phys. Rev. B 79, 153308 (2009).

${ }^{4}$ J. M. Tomczak, K. Haule, T. Miyake, A. Georges, and G. Kotliar, Phys. Rev. B 82, 085104 (2010).

${ }^{5}$ A. Bentien, G. K. H. Madsen, S. Johnsen, and B. B. Iversen, Phys. Rev. B 74, 205105 (2006)

${ }^{6}$ C. Petrovic, Y. Lee, T. Vogt, N. Dj. Lazarov, S. L. Bud'ko, and P. C. Canfield, Phys. Rev. B 72, 045103 (2005).

${ }^{7}$ C. Petrovic, J. W. Kim, S. L. Bud'ko, A. I. Goldman, P. C. Canfield, W. Choe, and G. J. Miller, Phys. Rev. B 67, 155205 (2003).

${ }^{8}$ A. V. Lukoyanov, V. V. Mazurenko, V. I. Anisimov, M. Sigrist, and T. M. Rice, Eur. Phys. J. B 53, 205 (2006).

${ }^{9}$ G. Mahan, B. Sales, and J. Sharp, Phys. Today 50(3), 42 (1997).

${ }^{10} \mathrm{P}$. Sun, N. Oeschler, S. Johnsen, B. B. Iversen, and F. Steglich, Appl. Phys. Express 2, 091102 (2009).

${ }^{11}$ P. Sun, N. Oeschler, S. Johnsen, B. B. Iversen, and F. Steglich, Dalton Trans. 39, 1012 (2010).

${ }^{12}$ R. Hu, V. F. Mitrović, and C. Petrovic, Phys. Rev. B 79, 064510 (2009).

${ }^{13}$ T. Harada, J. Phys. Soc. Jpn. 67, 1352 (1998).

${ }^{14}$ K. Behnia, D. Jaccard, and J. Flouquet, J. Phys.: Condens. Matter 16 5187 (2004).

${ }^{15}$ K. C. Sood and G. S. Verma, Phys. Rev. B 5, 3165 (1972).

${ }^{16} \kappa_{L}\left(=\kappa-\kappa_{e}\right)$ is estimated based on the Wiedemann-Franz law $\rho \kappa_{e} / T=L_{0}$, with the Lorenz number $L_{0}=2.44 \times 10^{-8} \mathrm{~V}^{2} / \mathrm{K}^{2}$.

${ }^{17}$ Y. Sun, S. Johnsen, P. Eklund, M. Sillassen, J. Bøttiger, N. Oeschler, P. Sun, F. Stgelich, and B. B. Iversen, J. Appl. Phys. 106, 033710 (2009).

${ }^{18}$ Y. Sun, E. Zhang, S. Johnsen, M. Sillassen, P. Sun, F. Steglich, J. Bøttiger, and B. B. Iversen, J. Phys. D: Appl. Phys. 43, 205402 (2010). 